\title{
Performance of Wideband CDMA Using Space-Time Spreading over Multipath Nakagami Fading Channels
}

\author{
Lie-Liang Yang and Lajos Hanzo \\ Dept. of Electronics and Computer Sciences \\ University of Southampton, SO17 1BJ, UK. \\ Tel: +44-703-593 125, Fax: +44-703-594 508
}

\begin{abstract}
In this contribution the performance of wideband code-division multiple-access (W-CDMA) systems using spacetime spreading (STS) based transmit diversity is investigated, when frequency-selective Nakagami- $m$ fading channels, multiuser interference and background noise are considered. The analysis and numerical results suggest that significant diversity gain can be achieved by employing several transmit antennas or/and by combining several multipath components. Furthermore, both the transmit diversity and the frequency-selective diversity appear to have the same order of importance.
\end{abstract}

\section{INTRODUCTION}

The capacity and the achievable data rate of wireless communication systems is limited by the time-varying characteristics of the channels. An efficient technique of combating the timevarying effects of wireless channels is employing diversity. In recent years, space-time coding has received much attention as an effective transmit diversity technique used for combating fading in wireless communications [1], [2]. Space-time block coding [2] assisted transmit diversity has now been adapted as an optional diversity mode in the third-generation (3G) wireless systems known as IMT2000 using wideband code-division multiple-access (W-CDMA) [3], [4]. Inspired by space-time codes, in [5] an attractive transmit diversity scheme based on space-time spreading (STS) has been proposed for employment in CDMA systems. More specifically, a STS scheme designed for supporting two transmission antennas and one receiver antenna has been included in the cdma2000 W-CDMA standard [4]. In [5] the performance of CDMA systems using STS has been investigated, when the channel is modeled either as a flat or as a frequency-selective Rayleigh fading channel in the absence of multiuser interference. It was argued that the proposed STS scheme is capable of attaining the maximal achievable transmit diversity gain without using extra spreading codes and without an increased transmit power. Furthermore, the results recorded for transmission over frequency-selective Rayleigh fading channels (Fig.4 of [5]) show that when there is a sufficiently high number of resolvable paths, a CDMA system using a single transmit antenna and a conventional RAKE receiver is capable of achieving an adequate diversity gain.

Wideband CDMA channels are typically frequency- selective fading channels, having a number of resolvable paths. Therefore, in this contribution, we investigate the performance

This work has been funded in the framework of the IST project IST-199912070 TRUST, which is partly funded by the European Union. The authors would like to acknowledge the contributions of their colleagues. of W-CDMA systems using STS based transmit diversity, when encountering multipath Nakagami- $m$ fading channels, multiuser interference and background noise. A Bit Error Ratio (BER) expression is derived, when Gaussian approximation [6] of the multiuser interference and that of the multipath interference is invoked. The analysis and the numerical results show that both the STS and the frequency-selectivity of the channel appear to have the same order of importance, especially, when the power decay factor of the multipath intensity profile (MIP) [7] is low.

\section{SYSTEM MODEL}

\section{A. Transmitted Signal}

The W-CDMA system considered in this paper consists of $U$ transmitter antennas and one receiver antenna. The transmitter schematic of the $k$ th user and the receiver schematic of the reference user are shown in Fig.1, where real-valued data symbols using BPSK modulation and real-valued spreading [5] were assumed. As shown in Fig.1(a), at the transmitter side the binary input data stream having a bit duration of $T_{b}$ is serial-to-parallel (S/P) converted to $U$ parallel sub-streams. The new bit duration of each parallel sub-stream, in other words the symbol duration becomes $T_{s}=U T_{b}$. After $\mathrm{S} / \mathrm{P}$ conversion, the $U$ number of parallel bits are direct-sequence spread using the STS schemes proposed in [5] with the aid of $U$ number of orthogonal spreading sequences - for example Walsh codes - having a period of $U G$, where $G=T_{b} / T_{c}$ represents the number of chips per bit and $T_{c}$ is the chip-duration of the orthogonal spreading sequences. As seen in Fig.1(a), following STS, the $U$ parallel signals to be mapped to the $U$ transmission antennas are scrambled using the $k$ th user's pseudo-noise $(\mathrm{PN})$ sequence $\mathrm{PN}_{k}(t)$, in order that the transmitted signals become randomized, and to ensure that the orthogonal spreading sequences employed within the STS block of Fig.1 can be reused by the other users. Finally, after the PN sequence based scrambling, the $U$ number of parallel signals are carrier modulated and transmitted by the corresponding $U$ number of antennas.

As described above, we have assumed that the number of parallel data sub-streams, the number of orthogonal spreading sequences used by the STS block of Fig.1 and the number of transmission antennas is the same, namely $U$. This specific STS scheme constitutes a specific sub-class of the generic family of STS schemes, where the number of parallel data sub-streams, the number of orthogonal spreading sequences required by STS 
block and the number of transmission antennas may take different values. The study conducted in [5] has shown that the number of orthogonal spreading sequences required by STS is usually higher, than the number of parallel sub-streams. The STS scheme having an equal number of parallel sub-streams, orthogonal STS-related spreading sequences as well as transmission antennas constitutes an attractive scheme, since this STS scheme is capable of providing maximal transmit diversity without requiring extra spreading codes for STS. Note that for the specific values of $U=2,4$ the above mentioned attractive STS schemes have been specified in [5]. In this contribution, we only investigate these attractive STS schemes.

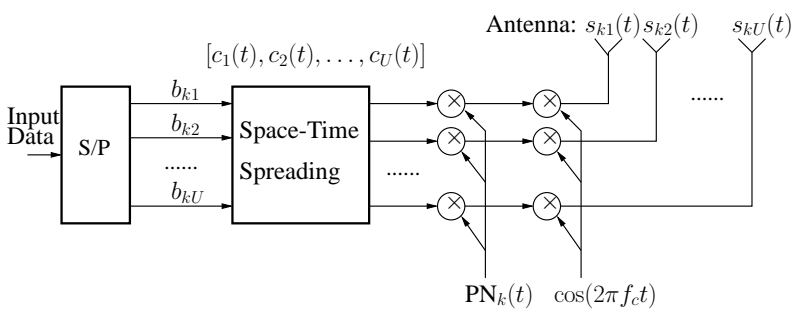

(a) Transmitter

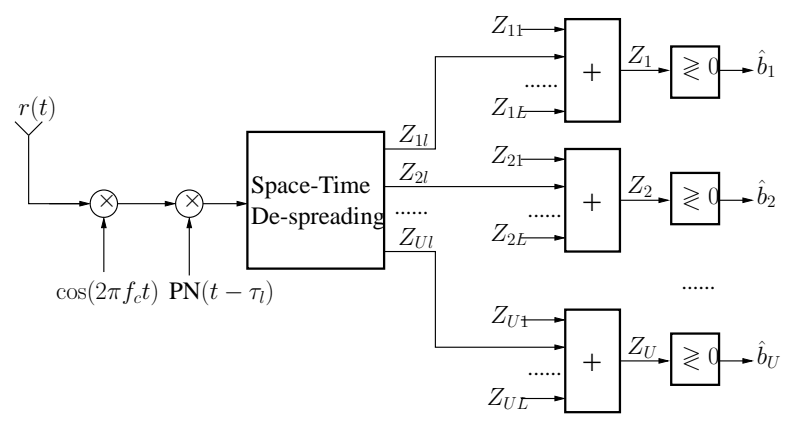

(b) Receiver

Fig. 1. Transmitter and receiver block diagram of the W-CDMA system using space-time spreading.

Based on the philosophy of STS as discussed in [5] and referring to Fig.1(a), the transmitted signal of the $k$ th user can be expressed as

$$
\mathbf{s}_{k}(t)=\sqrt{\frac{2 P}{U^{2}}} \mathbf{c}(t) \mathbf{B}_{U}(t) \times \mathrm{PN}_{k}(t) \cos \left(2 \pi f_{c} t\right),
$$

where $P$ represents each user's transmitted power, which is constant for all users, $\mathbf{s}_{k}(t)=\left[s_{k 1}(t) s_{k 2}(t) \ldots s_{k U}(t)\right]$ represents the transmitted signal vector of the $U$ transmission antennas, while $\mathrm{PN}_{k}(t)$ and $f_{c}$ represent the DS scrambling based spreading waveform and the subcarrier frequency, respectively. The scrambling sequence waveform is given by $\mathrm{PN}_{k}(t)=\sum_{j=-\infty}^{\infty} p_{k j} P_{T_{c}}\left(t-j T_{c}\right)$, where $p_{k j}$ assumes values of +1 or -1 with equal probability, while $P_{T_{c}}(t)$ is the rectangular chip waveform, which is defined over the interval $\left[0, T_{c}\right)$. In (1) the vector $\mathbf{c}(t)=\left[c_{1}(t) c_{2}(t) \ldots c_{U}(t)\right]$ is constituted by the $U$ number of orthogonal signals assigned for the STS, $c_{i}(t)=\sum_{j=-\infty}^{\infty} c_{i j} P_{T_{c}}\left(t-j T_{c}\right), i=1,2, \ldots, U$ denotes the individual components of the STS-based orthogonal spread signals, where $\left\{c_{i j}\right\}$ is an orthogonal sequence of period $U G$ for each index $i$; $\mathbf{B}_{U}(t)$ represents the $U \times U$ dimensional transmitted data matrix created by mapping $U$ input data bits to the $U$ parallel sub-streams according to specific design rules [5], so that the maximum possible transmit diversity is achieved, while using relatively low-complexity signal detection algorithms. Specifically, $\mathbf{B}_{U}(t)$ can be expressed as

$$
\mathbf{B}_{U}(t)=\left(\begin{array}{cccc}
a_{11} b_{k, 11} & a_{12} b_{k, 12} & \ldots & a_{1 U} b_{k, 1 U} \\
a_{21} b_{k, 21} & a_{22} b_{k, 22} & \ldots & a_{2 U} b_{k, 2 U} \\
\vdots & \vdots & \ddots & \vdots \\
a_{U 1} b_{k, U 1} & a_{U 2} b_{k, U 2} & \ldots & a_{U U} b_{k, U U}
\end{array}\right)(t)
$$

where the time dependence of the $(i, j)$ th element is indicated at the right of the matrix for simplicity. In (2) $a_{i j}$ represents the sign of the element at the $i$ th row and the $j$ th column, which is determined by the STS design rule, while $b_{k, i j}$ is the data bit assigned to the $(i, j)$ th element, which is one of the $U$ input data bits $\left\{b_{k 1}, b_{k 1}, \ldots, b_{k U}\right\}$ of user $k$. Each input data bit of $\left\{b_{k 1}, b_{k 2}, \ldots, b_{k U}\right\}$ appears only once in any a given row and in any given column.

\section{B. Channel Model}

The $U$ number of parallel signal components $\mathbf{s}_{k}(t)=$ $\left[\begin{array}{llll}s_{k 1}(t) & s_{k 2}(t) & \ldots & s_{k U}(t)\end{array}\right]$ are transmitted by the $U$ number of antennas over frequency-selective fading channels, where each parallel signal component experiences independent frequencyselective Nakagami- $m$ fading. The complex low-pass equivalent representation of the impulse response experienced by the $u$ th parallel signal component of user $k$ is given by [7]

$$
h_{k}^{u}(t)=\sum_{l=1}^{L} h_{k l}^{u} \delta\left(t-\tau_{k l}\right) \exp \left(j \phi_{k l}^{u}\right),
$$

where $h_{k l}^{u}, \tau_{k l}$ and $\psi_{k l}^{u}$ represent the attenuation factor, delay and phase-shift of the $l$ th multipath component of the channel, respectively, while $L$ is the total number of resolvable multipath components and $\delta(t)$ is the Kronecker Delta-function. We assume that the phases $\left\{\psi_{k l}^{u}\right\}$ in (3) are independent identically distributed (iid) random variables uniformly distributed in the interval $[0,2 \pi)$, while the $L$ multipath attenuations $\left\{h_{k l}^{u}\right\}$ in (3) are independent Nakagami random variables with a Probability Density Function (PDF) of [6]

$$
\begin{aligned}
p\left(h_{k l}^{u}\right) & =M\left(h_{k l}^{u}, m_{k l}^{(u)}, \Omega_{k l}^{u}\right), \\
M(R, m, \Omega) & =\frac{2 m^{m} R^{2 m-1}}{\Gamma(m) \Omega^{m}} e^{(-m / \Omega) R^{2}},
\end{aligned}
$$

where $\Gamma(\cdot)$ represents the gamma function [7], and $m_{k l}^{(u)}$ is the Nakagami- $m$ fading parameter, which characterizes the severity of the fading over the $l$-th resolvable path between the $u$ th transmission antenna and user $k$. The parameter $\Omega_{k l}^{u}$ in (4) is the second moment of $h_{k l}^{u}$, i.e. we have $\Omega_{k l}^{u}=E\left[\left(\alpha_{k l}^{u}\right)^{2}\right]$. We assume a negative exponentially decaying multipath intensity profile (MIP) given by $\Omega_{k l}^{u}=\Omega_{k 1}^{u} e^{-\eta(l-1)}, \quad \eta \geq 0$, where $\Omega_{k 1}^{u}$ is the average signal strength corresponding to the first resolvable path and $\eta$ is the rate of average power decay. 
We support $K$ asynchronous CDMA users in the system and assume perfect power control. Consequently, when the $K$ users' signals obeying the form of (1) are transmitted over the frequency-selective fading channels characterized by (3), the received complex low-pass equivalent signal at a given mobile station can be expressed as

$$
\begin{aligned}
R(t)=\sum_{k=1}^{K} \sum_{l=1}^{L} & \sqrt{\frac{2 P}{U^{2}}} \mathbf{c}\left(t-\tau_{k l}\right) \mathbf{B}_{U}\left(t-\tau_{k l}\right) \mathbf{h}_{k l} \\
\times & \mathrm{PN}_{k}\left(t-\tau_{k l}\right)+N(t),
\end{aligned}
$$

where $N(t)$ is the complex valued low-pass-equivalent Additive White Gaussian Noise (AWGN) having a double-sided spectral density of $N_{0}$, while

$$
\mathbf{h}_{k l}=\left(\begin{array}{c}
h_{k l}^{1} \exp \left(j \psi_{k l}^{1}\right) \\
h_{k l}^{2} \exp \left(j \psi_{k l}^{2}\right) \\
\cdots \\
h_{k l}^{U} \exp \left(j \psi_{k l}^{U}\right)
\end{array}\right), \quad \begin{gathered}
k=1,2, \ldots, K \\
l=1,2, \ldots, L
\end{gathered}
$$

represents the channel's complex impulse response in the context of the $k$ th user and the $l$ th resolvable path, where $\psi_{k l}^{u}=$ $\phi_{k l}^{u}-2 \pi f_{c} \tau_{k l}$.

\section{Receiver Model}

Let the first user be the user-of-interest and consider a receiver using space-time de-spreading as well as diversity combining, as shown in Fig.1(b), where the subscript of the reference user's signal has been omitted for notational convenience. The receiver of Fig.1(b) carries out the inverse processing of Fig.1(a), in addition to multipath diversity combining. In Fig.1(b) the received signal is first down-converted using the carrier frequency $f_{c}$, and then de-scrambled using the DS scrambling sequence of $\operatorname{PN}\left(t-\tau_{l}\right)$ in the context of the $l$ th resolvable path, where we assumed that the receiver is capable of achieving near-perfect multipath-delay estimation for the reference user. The de-scrambled signal associated with the $l$ th resolvable path is space-time de-spread using the approach of [5], in order to obtain $U$ separate variables, $\left\{Z_{1 l}, Z_{2 l}, \ldots, Z_{U l}\right\}$, corresponding to the $U$ parallel data bits $\left\{b_{1}, b_{2}, \ldots, b_{U}\right\}$, respectively. Following space-time despreading, a decision variable is formed for each parallel transmitted data bit of $\left\{b_{1}, b_{2}, \ldots, b_{U}\right\}$ by combining the corresponding variables associated with the $L$ number of resolvable paths, which can be expressed as

$$
Z_{u}=\sum_{l=1}^{L} Z_{u l}, u=1,2, \ldots, U .
$$

Finally, the $U$ number of transmitted data bits $\left\{b_{1}, b_{2}, \ldots, b_{U}\right\}$ can be decided based on the decision variables $\left\{Z_{u}\right\}_{u=1}^{U}$ using the conventional decision rule of a BPSK scheme.

\section{Detection of Space-Time Spread W-CDMA SIGNALS}

Let $\mathbf{d}_{l}=\left[\begin{array}{llll}d_{1 l} & d_{2 l} & \ldots & d_{U l}\end{array}\right]^{T}, l=1,2, \ldots, L$ - where $T$ denotes vector transpose - represent the correlator's output variable vector in the context of the $l$ th $(l=1,2, \ldots, L)$ resolvable path, where

$$
d_{u l}=\int_{\tau_{l}}^{U T_{b}+\tau_{l}} R(t) c_{u}\left(t-\tau_{l}\right) \operatorname{PN}\left(t-\tau_{l}\right) d t .
$$

When substituting (5) into (8), it can be shown that

$$
\begin{aligned}
d_{u l} & =\sqrt{2 P} T_{b}\left[a_{u 1} b_{u 1} h_{l}^{1} \exp \left(j \psi_{l}^{1}\right)+a_{u 2} b_{u 2} h_{l}^{2} \exp \left(j \psi_{l}^{2}\right)\right. \\
& \left.+\ldots+a_{u U} b_{u U} h_{l}^{U} \exp \left(j \psi_{l}^{U}\right)\right]+J_{u}(l)
\end{aligned}
$$

for $u=1,2, \ldots, U$, where

$$
J_{u}(l)=J_{S u}(l)+J_{M u}(l)+N_{u}(l), u=1,2, \ldots, U
$$

and $J_{S u}(l)$ is due to the multipath-induced self-interference of the signal-of-interest inflicted upon the $l$ th path signal, where $J_{S u}(l)$ can be expressed as

$$
\begin{array}{r}
J_{S u}(l)=\sum_{j=1, j \neq l}^{L} \sqrt{\frac{2 P}{U^{2}}} \int_{\tau_{l}}^{U T_{b}+\tau_{l}} \mathbf{c}\left(t-\tau_{j}\right) \mathbf{B}_{U}\left(t-\tau_{j}\right) \mathbf{h}_{j} \\
\times \operatorname{PN}\left(t-\tau_{j}\right) c_{u}\left(t-\tau_{l}\right) \operatorname{PN}\left(t-\tau_{l}\right) d t
\end{array}
$$

while $J_{M u}(l)$ represents the multiuser interference due to the signals transmitted simultaneously by the other users, which can be expressed as

$$
\begin{aligned}
J_{M u}(l)=\sum_{k=2}^{K} & \sum_{j=1}^{L} \sqrt{\frac{2 P}{U^{2}}} \int_{\tau_{l}}^{U T_{b}+\tau_{l}} \mathbf{c}\left(t-\tau_{k j}\right) \mathbf{B}_{U}\left(t-\tau_{k j}\right) \\
& \times \mathbf{h}_{k j} \operatorname{PN}_{k}\left(t-\tau_{k j}\right) c_{u}\left(t-\tau_{l}\right) \operatorname{PN}\left(t-\tau_{l}\right) d t .
\end{aligned}
$$

Finally $N_{u}(l)$ is due to the AWGN, which can be written as

$$
N_{u}(l)=\int_{\tau_{l}}^{U T_{b}+\tau_{l}} N(t) c_{u}\left(t-\tau_{l}\right) \operatorname{PN}\left(t-\tau_{l}\right) d t,
$$

which is a Gaussian distributed variable having zero mean and a variance of $2 U N_{0} T_{b}$.

Let $\mathbf{J}(l)=\left[J_{1}(l) J_{2}(l) \ldots J_{U}(l)\right]^{T}$. Then, the correlator's output variable vector $\mathbf{d}_{l}$ can be expressed as

$$
\mathbf{d}_{l}=\sqrt{2 P} T_{b} \mathbf{B}_{U} \mathbf{h}_{l}+\mathbf{J}(l), l=1,2, \ldots, L,
$$

where $\mathbf{B}_{U}$ is the reference user's $U \times U$-dimensional transmitted data matrix, which is given by (2), but ignoring the time dependence, while $\mathbf{h}_{l}$ is the channel's complex impulse response between the base station and the reference user, as shown in (6) in the context of the reference user.

Attractive STS schemes have the property [5] of $\mathbf{B}_{U} \mathbf{h}_{l}=$ $\mathbf{H}_{U}$ b, i.e., Equation (14) can be written as

$$
\mathbf{d}_{l}=\sqrt{2 P} T_{b} \mathbf{H}_{U} \mathbf{b}+\mathbf{J}(l),
$$

where $\mathbf{b}=\left[\begin{array}{llll}b_{1} & b_{2} & \ldots & b_{U}\end{array}\right]^{T}$ represents the $U$ number of transmitted data bits, while $\mathbf{H}_{U}$ is a $U \times U$-dimensional matrix with elements from $\mathbf{h}_{l}$. Each element of $\mathbf{h}_{l}$ appears once and only 
once in a given row and also in a given column of the matrix $\mathbf{H}_{U}$ [5]. The matrix $\mathbf{H}_{U}$ can be expressed as

$$
\mathbf{H}_{U}(l)=\left(\begin{array}{cccc}
\alpha_{11}(l) & \alpha_{12}(l) & \ldots & \alpha_{1 U}(l) \\
\alpha_{21}(l) & \alpha_{22}(l) & \ldots & \alpha_{2 U}(l) \\
\vdots & \vdots & \ddots & \vdots \\
\alpha_{U 1}(l) & \alpha_{U 2}(l) & \ldots & \alpha_{U U}(l)
\end{array}\right)
$$

where $\alpha_{i j}(l)$ takes the form of $d_{i j} h_{l}^{m} \exp \left(j \psi_{l}^{m}\right)$, and $d_{i j} \in$ $\{+1,-1\}$ represents the sign of the $(i, j)$ th element of $\mathbf{H}_{U}$, while $h_{l}^{m} \exp \left(j \psi_{l}^{m}\right)$ belongs to the $m$ th element of $\mathbf{h}_{l}$.

With the aid of the analysis in [5], it can be shown that the matrix $\mathbf{H}_{U}(l)$ has the property of $\operatorname{Re}\left\{\mathbf{H}_{U}^{\dagger}(l) \mathbf{H}_{U}(l)\right\}=$ $\mathbf{h}_{l}^{\dagger} \mathbf{h}_{l} \cdot \mathbf{I}$, where $\dagger$ denotes complex conjugate transpose and $\mathbf{I}$ represents a $U \times U$-dimensional unity matrix. Letting $\mathbf{h}_{u}(l)$ denote the $u$ th column of $\mathbf{H}_{U}(l)$, the variable $Z_{u l}$ in (7) can be expressed as [5]

$$
\begin{aligned}
Z_{u l}= & \operatorname{Re}\left\{\mathbf{h}_{u}^{\dagger}(l) \mathbf{d}_{l}\right\}=\sqrt{2 P} T_{b} b_{u} \sum_{u=1}^{U}\left|h_{l}^{u}\right|^{2} \\
& +\operatorname{Re}\left\{\mathbf{h}_{u}^{\dagger}(l) \mathbf{J}(l)\right\}, u=1,2, \ldots, U .
\end{aligned}
$$

Finally, according to (7) the decision variables associated with the $U$ parallel transmitted data bits $\left\{b_{1}, b_{2}, \ldots, b_{U}\right\}$ of the reference user can be expressed as

$$
Z_{u}=\sqrt{2 P} T_{b} b_{u} \sum_{l=1}^{L} \sum_{u=1}^{U}\left|h_{l}^{u}\right|^{2}+\sum_{l=1}^{L} \operatorname{Re}\left\{\mathbf{h}_{u}^{\dagger}(l) \mathbf{J}(l)\right\},
$$

which shows that the receiver is capable of achieving a diversity order of $U L$, as indicated by the related sums of the first term.

With the aid of the Gaussian approximation and after some arduous analysis, it can be shown that the average BER of the STS-assisted W-CDMA system using $U$ transmission antennas can be expressed as

$$
P_{b}(E)=\frac{1}{\pi} \int_{0}^{\pi / 2} \prod_{l=1}^{L} \prod_{u=1}^{U}\left(\frac{m_{l}^{(u)} \sin ^{2} \theta}{\bar{\gamma}_{l u}+m_{l}^{(u)} \sin ^{2} \theta}\right)^{m_{l}^{(u)}} d \theta
$$

where

$$
\begin{array}{r}
\bar{\gamma}_{l u}=\frac{1}{U}\left[\frac{(2 K+1) q(L, \eta)-3}{3 G}+\left(\frac{\Omega_{1} E_{b}}{N_{0}}\right)^{-1}\right]^{-1} \\
\times e^{-\eta(l-1)} .
\end{array}
$$

Again, (19) shows that the diversity order achieved is $L U$.

\section{NUMERICAL RESULTS}

In Figs. 2 - 5 we compare the BER performance of the STSassisted W-CDMA system transmitting over flat-fading channels and that of the conventional RAKE receiver using only one transmission antenna, but communicating over frequencyselective fading channels. The results in these figures were all

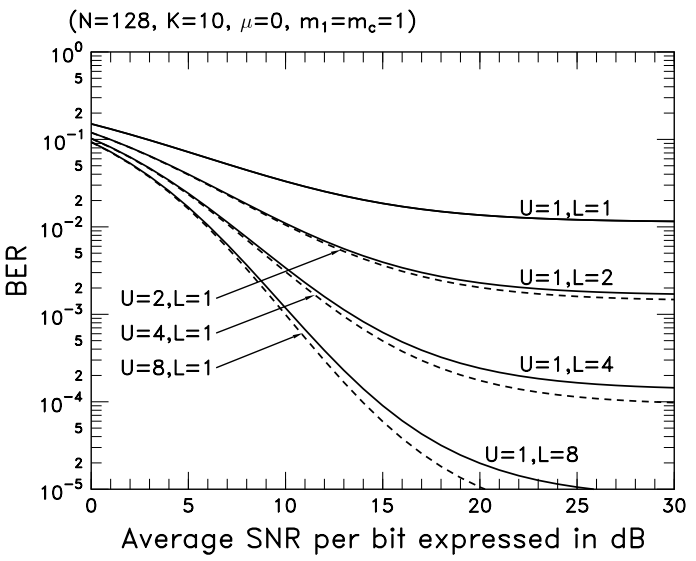

Fig. 2. BER versus the SNR per bit, $E_{b} / N_{0}$, performance comparison between the space-time spreading based transmit diversity scheme and the conventional RAKE receiver arrangement using only one transmission antenna when communicating over flat-fading (for space-time spreading) and multipath ( for RAKE) Rayleigh fading $\left(m_{l}=m_{c}=1\right.$ ) channels by assuming that the average power decay rate was $\eta=0$.



Fig. 3. BER versus the SNR per bit, $E_{b} / N_{0}$, performance comparison between the space-time spreading based transmit diversity scheme and the conventional RAKE receiver arrangement using only one transmission antenna when communicating over flat-fading (for space-time spreading) and multipath (for RAKE) Nakagami- $m$ fading channels by assuming that the average power decay rate was $\eta=0.2$, where $m_{1}=2$ indicates that the first resolvable path constitutes a moderately fading path, while the other resolvable paths experience more severe Rayleigh fading $\left(m_{c}=1\right)$. 
evaluated from (19) by assuming appropriate parameters, which are explicitly shown in the corresponding figures. In Figs. 2 and 3 the BER was drawn against the SNR/bit, namely $E_{b} / N_{0}$, while in Figs. 4 and 5 the BER was drawn against the number of users, $K$, supported by the system. From the results we observe that for transmission over Rayleigh fading channels $\left(m_{l}=1\right)$, as characterized by Figs. 2 and 4, both the STS based transmit diversity scheme transmitting over the frequency non-selective Rayleigh fading channel and the conventional RAKE receiver scheme communicating over frequency-selective Rayleigh fading channels having the same number of resolvable paths as the number of transmission antennas in the STS-assisted scheme achieved a similar BER performance, with the STS scheme slightly outperforming the conventional RAKE scheme. For transmission over general Nakagami- $m$ fading channels, if the first resolvable path is less severely faded, than the other resolvable paths, such as in Figs. 3 and 5 where $m_{1}=2$ and $m_{2}=m_{3}=\ldots=m_{c}=1$, the STS based transmit diversity scheme communicating over the frequency non-selective Rayleigh fading channel may significantly outperform the corresponding conventional RAKE receiver assisted scheme communicating over frequency-selective Rayleigh fading channels. This is because the STS based transmit diversity scheme communicated over a single non-dispersive path, which benefitted from having a path experiencing moderate fading. However, if the number of resolvable paths is sufficiently high, the conventional RAKE receiver scheme is also capable of achieving a satisfactory BER performance.

\section{Conclusions}

In this contribution we have investigated the performance of STS-assisted W-CDMA systems, when multipath Nakagami$m$ fading, multiuser interference and background noise induced impairments are considered. Our analysis and numerical results demonstrated that significant diversity gain can be achieved by using several transmit antennas or/and by combining several multipath components. Furthermore, both the transmit diversity and the frequency-selective diversity have a similar influence on the BER performance of the W-CDMA systems considered.

\section{REFERENCES}

[1] V. Tarokh, N. Seshadri, and A. R. Calderbank, "Space-time codes for high data rate wireless communication: performance criterion and code construction," IEEE Transactions on Information Theory, vol. 44, pp. 744765, March 1998.

[2] V. Tarokh, H. Jafarkhani, and A. R. Calderbank, "Space-time block coding for wireless communications: performance results," IEEE Journal on Selected Areas in Communications, vol. 17, pp. 451-460, March 1999.

[3] Proposed TDOC: 662/98 to ETSI SMG2 UMTS Standards, Space-time block coded transmit antenna diversity for WCDMA, December 1998.

[4] Telcomm. Industry Association (TIA), TIA/EIA Interim Standard: Physical Layer Standard for cdma2000 Standards for Spread Spectrum Systems, 2000.

[5] B. Hochwald, T. L. Marzetta, and C. B. Papadias, "A transmitter diversity scheme for wideband CDMA systems based on space-time spreading," IEEE Journal on Selected Areas in Communications, vol. 19, pp. 48-60, January 2001.

[6] T. Eng and L. B. Milstein, "Coherent DS-CDMA performance in Nakagami multipath fading," IEEE Transactions on Communications, vol. 43, pp. 1134-1143, Feb./Mar./Apr. 1995.

[7] J. G. Proakis, Digital Communications. McGraw Hill, 3rd ed., 1995.

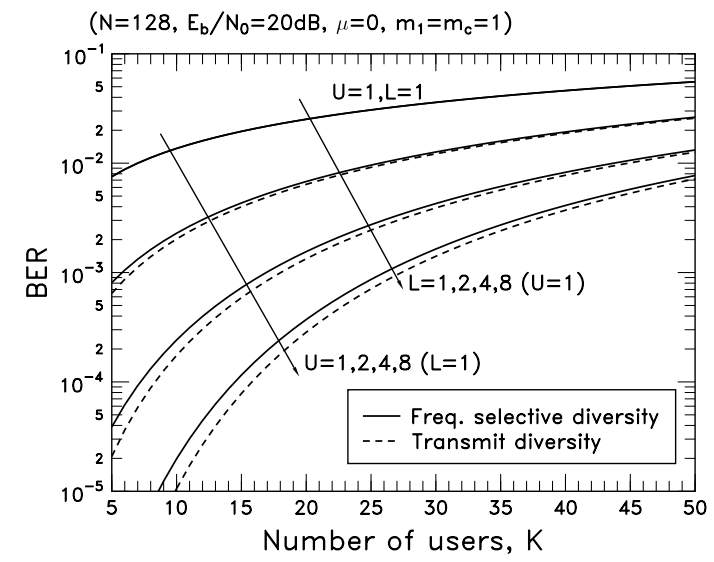

Fig. 4. BER versus the number of users, $K$, performance comparison between the space-time spreading based transmit diversity scheme and the conventional RAKE receiver arrangement using only one transmission antenna when communicating over flat-fading (for space-time spreading) and multipath (for RAKE) Rayleigh fading channels by assuming that the average power decay rate was $\eta=0$.



Fig. 5. BER versus the number of users, $K$, performance comparison between the space-time spreading based transmit diversity scheme and the conventional RAKE receiver arrangement using only one transmission antenna when communicating over the flat-fading (for space-time spreading) and multipath (for RAKE) Nakagami- $m$ fading channels by assuming that the average power decay rate was $\eta=0.2$, where $m_{1}=2$ indicates that the first resolvable path constitutes a moderately fading path, while the other resolvable paths experience more severe Rayleigh fading $\left(m_{c}=1\right)$. 